\title{
Chaos Synchronization and Spontaneous Symmetry-Breaking in Symmetrically Delay-Coupled Semiconductor Lasers
}

\author{
Tilmann Heil, Ingo Fischer, and Wolfgang Elsässer \\ Institute of Applied Physics, Darmstadt University of Technology, Schloßgartenstrasse 7, D-64289 Darmstadt, Germany \\ Josep Mulet and Claudio R. Mirasso* \\ Instituto Mediterraneo de Estudios Avanzados, CSIC-UIB, Campus UIB, E-07071 Palma de Mallorca, Spain
}

(Received 5 June 2000)

\begin{abstract}
We present experimental and numerical investigations of the dynamics of two device-identical, optically coupled semiconductor lasers exhibiting a delay in the coupling. Our results give evidence for subnanosecond coupling-induced synchronized chaotic dynamics in conjunction with a spontaneous symmetry-breaking: we find a well-defined time lag between the dynamics of the two lasers, and an asymmetric physical role of the subsystems. We demonstrate that the leading laser synchronizes its lagging counterpart, whereas the synchronized lagging laser drives the coupling-induced instabilities.
\end{abstract}

DOI: 10.1103/PhysRevLett.86.795

Coupled nonlinear oscillators exhibit a great variety of fundamental dynamical phenomena including synchronization of periodic and even chaotic oscillations. These phenomena are encountered in many different systems in nature and science ranging from chemical oscillators, population dynamics, physiological interactions, coupled neurons, to mechanical oscillators and lasers; see, e.g., [1]. Thus, the understanding of the dynamics of nonlinear coupled oscillators is essential for a wide range of scientific investigations. In real systems, the coupling often exhibits a significant delay due to the separation between the subsystems. The additional degrees of freedom present in these delay-coupled oscillator systems may qualitatively alter their dynamical behavior. Experimental studies have not yet addressed the impact of delay on the dynamics of nonlinear coupled oscillators, whereas theoretical investigations have already demonstrated bistability between synchronized and incoherent states [2] and stochastic resonance [3]. In order to study the dynamics of nonlinear delay-coupled oscillators experimentally, we have selected two distanced semiconductor lasers (SL) which are coupled via the optical field. The delay is determined by the propagation between the subsystems and is much larger than the SL internal oscillation periods. Recent studies of very weakly coupled SLs demonstrating localized synchronization of periodic oscillations [4] have underlined the fundamental relevance of this system. Furthermore, SLs have an immense potential for practical applications, in particular, for future telecommunications technologies: the proposal of novel communication systems using chaotic carriers based on chaos synchronization of distanced lasers $[5,6]$ has further boosted the interest in coupling and synchronization phenomena in SLs.

A scheme of our experimental setup is depicted in Fig. 1. The system consists of two uncoated Hitachi HLP1400 Fabry-Perot SLs. We have selected two device-identical lasers (optical spectra agree within $0.1 \mathrm{~nm}$, slope efficiency within $2 \%$, and threshold current within $5 \%$ ) produced
PACS numbers: 05.45.Xt, 42.55.Px, 42.65.Sf

from the same wafer in order to achieve the highest possible degree of symmetry. Both lasers are pumped at a constant level, upon which, optionally, a sinusoidal modulation may be superposed. The temperature of each laser is stabilized to better than $0.01 \mathrm{~K}$ and selected such that the frequencies of the two lasers match with an accuracy better than $1 \mathrm{GHz}$. Guaranteed by the polarizer (Pol.), the lasers are coherently coupled via the dominant TE component of the optical field. The neutral density filter (NDF) controls the coupling strength. The coupling time $\tau$ is determined by the propagation of the light between the lasers and has been varied between 3.8 and 5 ns. Thus, $\tau$ is significantly larger than the SL internal time scales, i.e., the relaxation oscillations, or the laser cavity round-trip resonance. We detect the intensity dynamics of both lasers simultaneously with dc to a $6 \mathrm{GHz}$ bandwidth and analyze the signal using a fast oscilloscope of a $3 \mathrm{GHz}$ analog bandwidth and an electrical spectrum analyzer (ESA). Thus, our detection equipment allows us to record the intensity time series of both lasers simultaneously on a subnanosecond time scale. In addition, we monitor the optical spectra of the two lasers with an optical spectrum analyzer (OSA) with a $0.1 \mathrm{~nm}$ resolution and detect the time averaged output power.

In the experiments, we inject a well-controlled amount of the emitted light of either laser into the other laser,

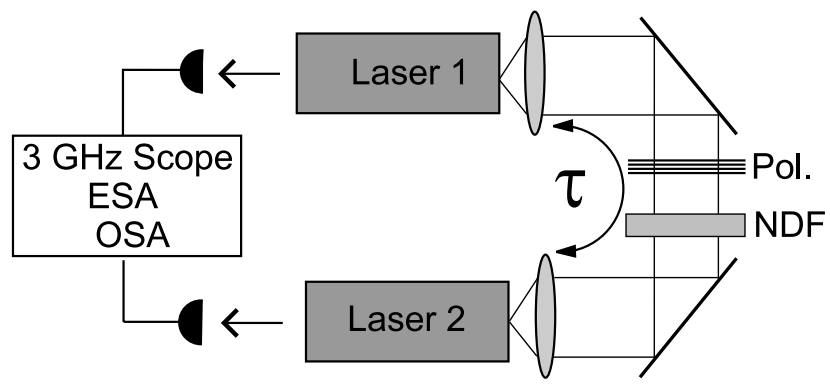

FIG. 1. Scheme of the experimental setup. 
approximately $5 \%$ of the respective output power, leading to a threshold reduction of $6 \%$ in both lasers. Pumping both lasers at their solitary threshold $I_{\mathrm{th}}^{\text {sol }}$, we observe striking coupling-induced instabilities: Fig. 2 depicts a $200 \mathrm{~ns}$ snapshot of both intensity time series of the coupled lasers; the lower trace is plotted inverted to ease the comparison between the two signals. The coupling-induced instabilities are characterized by fast intensity fluctuations on a subnanosecond time scale combined with pronounced intensity dropouts occurring on much slower time scales. The very similar rf spectra of both lasers exhibit a dominant low frequency part and peaks at frequencies corresponding to multiples of $1 /(2 \tau)$; the optical spectra display several longitudinal modes of the solitary laser. We note that this dynamical behavior closely compares to the low frequency fluctuations (LFF) phenomenon of a single SL subject to delayed optical feedback [7]. However, we emphasize that the instabilities in the present experiment are not induced by passive feedback due to external reflections. Starting from the situation depicted in Fig. 2, we reduce the injection current of one of the lasers below its transparency point. We observe that the other laser (still driven at its solitary threshold) exhibits stable emission, and, accordingly, a flat rf spectrum. Thus, the instabilities depicted in Fig. 2 indeed result from the coherent coupling with a delay between the two lasers.

In addition, Fig. 2 exhibits another remarkable dynamical phenomenon. The low frequency intensity dropouts always occur strongly correlated in both lasers, however, with a constant time lag between the two signals. This delay between the leading laser (leader) and the lagging laser (laggard) which exactly corresponds to the coupling time $\tau$ is the manifestation of a spontaneous symmetrybreaking in the system. To resolve the underlying fast dynamics, Fig. 3 presents a 9 ns zoom of the two noninverted intensity time series. For clarity, the laggard time series is shifted vertically and horizontally compensating

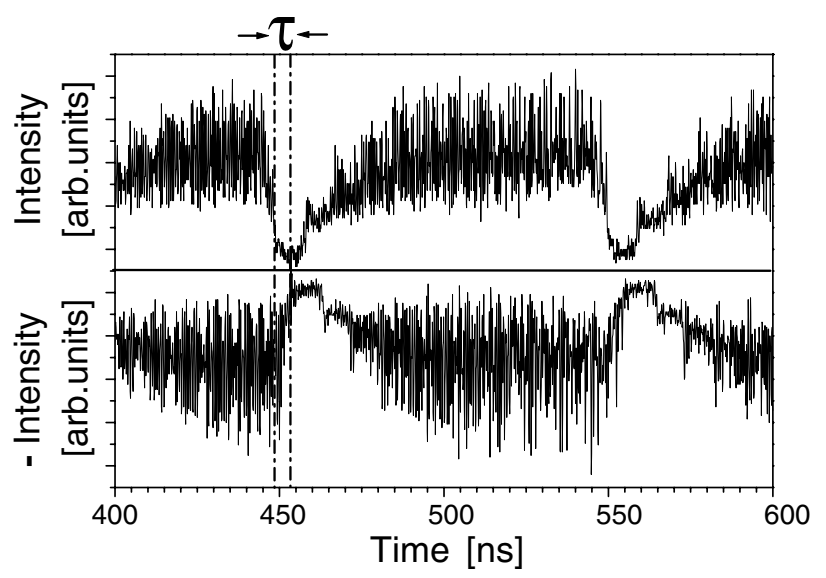

FIG. 2. Intensity time series of the two lasers. The lower trace shows the inverted time series. Both lasers are pumped at $I=I_{\mathrm{th}}^{\text {sol }}$. The threshold reduction due to the coupling amounts to $6 \%$. The coupling time is $\tau=4.75 \mathrm{~ns}$. the time delay $\tau$. Figure 3 demonstrates that both intensity and oscillation phase of the irregular dynamics of the two delay-coupled SLs are synchronous on a subnanosecond time scale. Furthermore, the rf and the optical spectra of leader and laggard are experimentally indistinguishable confirming the synchronization. By correlation analysis of a large number of pairs of time series, we confirm that the synchronization and the delay are robust against reasonable variations of the injection current, the coupling strength, and the coupling time; the delay between leader and laggard remains equal to the coupling time $\tau$. We note that previous research has already demonstrated spontaneous symmetry-breaking in coupled chaotic systems without delay [8]. However, what we observe here is a leaderlaggard-type conjunction of spontaneous symmetrybreaking with chaos synchronization which is linked to the delay present in the coupling.

We find that the difference between the solitary laser wavelengths is an easily accessible and well-defined control parameter determining the direction of the symmetry-breaking: always the laser detuned to higher energies takes the leader role. About $1 \mathrm{GHz}$ of detuning is already sufficient to create a well-defined leader-laggard configuration. In addition, we can interchange the dynamical role of the lasers in a symmetrical way by reversing the detuning. This behavior may be related to the asymmetric injection locking properties [9]. For zero detuning, finally, a spontaneous symmetry-breaking occurs, and one of the lasers is taking the lead by chance. In the experiment, we can achieve only a finite accuracy with respect to detuning and similarity between the subsystems. Therefore, we perform numerical simulations of two identical lasers in order to exclude any relevant influence of slight dissimilarities eventually present in the experiment.

For our numerical simulations, we have extended the fundamental SL rate equations for the slowly varying complex electrical field amplitudes $E_{1}, E_{2}$ and the carrier numbers $N_{1}, N_{2}$ by adding delay coupling terms $\kappa E_{2,1}(t-\tau)$ :

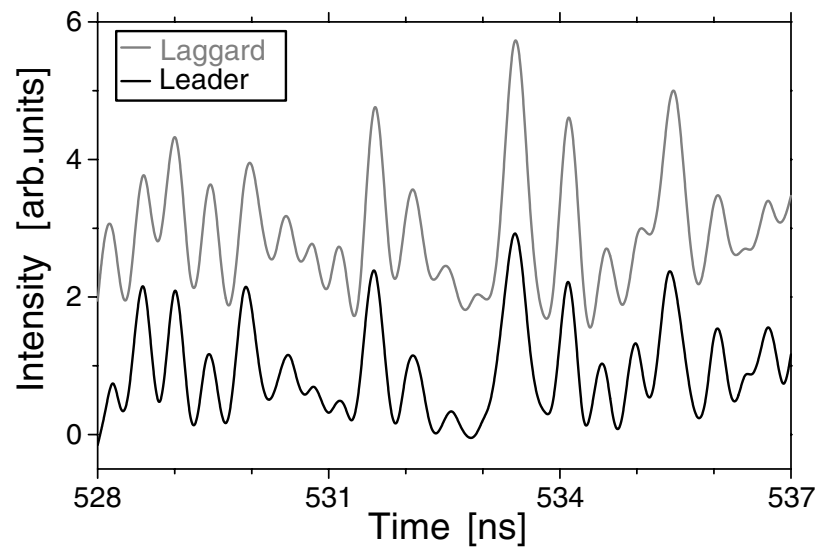

FIG. 3. 9 ns zoom of the intensity time series plotted in Fig. 2. Here, the time series of the laggard is noninverted, but shifted by $4.75 \mathrm{~ns}$ in time. 


$$
\begin{aligned}
\dot{E}_{1,2}(t)= & \frac{1}{2}(1+i \alpha)\left[G_{1,2}-\gamma\right] E_{1,2}+\kappa E_{2,1}(t-\tau) \\
& +\sqrt{2 \beta N_{1,2}} \xi_{1,2} \\
\dot{N}_{1,2}= & \frac{I}{q}-\gamma_{e} N_{1,2}-G_{1,2}\left|E_{1,2}\right|^{2} \\
G_{1,2}= & \frac{g\left(N_{1,2}-N_{t}\right)}{1+\varepsilon\left|E_{1,2}\right|^{2}}
\end{aligned}
$$

We assume zero detuning; i.e., both lasers emit at the same frequency. The parameters used in our numerical simulations correspond to the experimental conditions. We select identical parameters for both lasers noting that we obtain similar results even for slight differences between the lasers. The linewidth enhancement factor is $\alpha=3.5$, the cavity losses $\gamma=282 \mathrm{~ns}^{-1}$, and the differential gain $g=3.2 \times 10^{-6} \mathrm{~ns}^{-1}$. The coupling rate $\kappa=23 \mathrm{~ns}^{-1}$ accounts for the symmetrical coupling strength between the lasers. The coupling time is $\tau=4.75 \mathrm{~ns}$, the carrier decay rate $\gamma_{e}=1.66 \mathrm{~ns}^{-1}$, the carrier value at transparency $N_{t}=1.5 \times 10^{8}$, the gain saturation parameter $\varepsilon=5 \times 10^{-7}$, the current $I=I_{\mathrm{th}}^{\mathrm{sol}}$, and the spontaneous emission rate $\beta=10^{-6} \mathrm{~ns}^{-1}$. The spontaneous emission processes are modeled by including white Gaussian noise $\xi_{1,2}$ with zero mean and correlation $\left\langle\xi_{i}^{*}(t) \xi_{j}\left(t^{\prime}\right)\right\rangle=2 \delta_{i, j} \delta\left(t-t^{\prime}\right)$.

Figure 4 depicts a thus obtained numerical time series. Despite the simplicity of the used rate equation model, the numerical simulations are in excellent agreement with the experiment and capture the observed dynamical phenomena of delay-coupled SLs: the synchronized fast chaotic dynamics, the low frequency fluctuations, and the spontaneous symmetry-breaking appearing as a time lag of $\tau$ between the signals of the two lasers. The occurrence of the symmetry-breaking in the simulations is remarkable because equations and parameters are absolutely symmetric under exchange of the lasers. Indeed, in noiseless numeri-

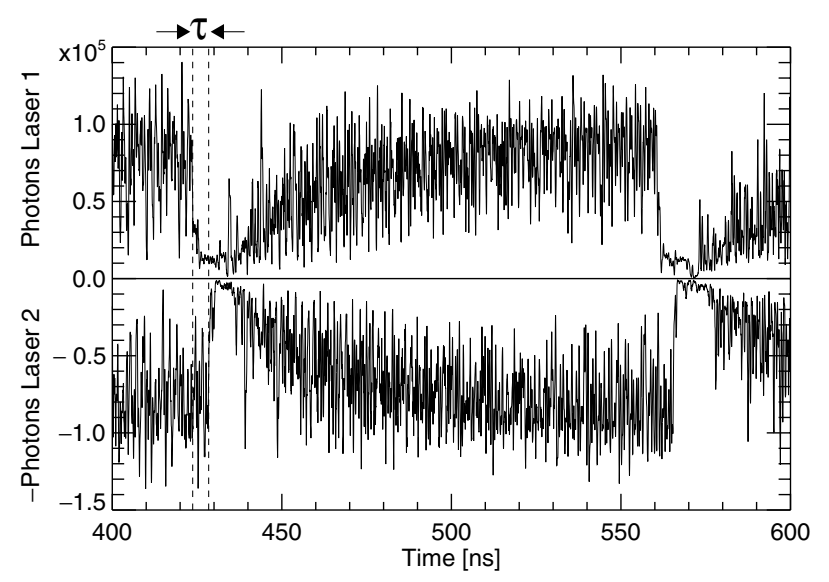

FIG. 4. Numerical intensity time series for two identical delay coupled SLs. The parameters correspond to the experiment shown in Fig. 2. cal simulations, starting both lasers from the same initial conditions, we find that the lasers evolve in a synchronized state without a time lag between the signals. However, the noise term or any small external perturbation breaks this symmetry. The zero-lag solution is lost, and the system exhibits the synchronized leader-laggard dynamics instead. We conclude that the zero-lag solution is unstable, and spontaneous emission prevents the system to operate in such a state.

So far, we have observed that the symmetry-breaking appears as a time lag of $\tau$ between the signals of the two lasers. For the physical understanding of the system it is essential to know whether there is also a change in the physical role of the subsystems associated with the symmetry-breaking, i.e., whether leader and laggard play a different role for the dynamics of the system, and what role this is. Accordingly, in the following experiment, we apply a small perturbation, a weak sinusodial modulation of $0.7 \% \mathrm{I}_{\mathrm{th}}^{\mathrm{sol}}$, to one of the lasers and investigate the effect on its unperturbed counterpart. The lasers are slightly detuned by $2.5 \mathrm{GHz}$ to guarantee a well-defined leaderlaggard configuration. If leader and laggard exhibit genuine chaos synchronization, the synchronized laggard should exhibit chaos pass filtering properties [6]. This peculiar property of synchronized systems contrasts them to linear amplifiers: if a perturbation is modulated on a chaotic signal and sent to a receiver system, synchronization leads to the fact that the receiver synchronizes only to the chaotic signal but suppresses the perturbation. In contrast, a linear amplifier receives both chaotic signal and perturbation in the same way.

Figure 5 depicts the rf spectra of the two delay-coupled lasers: (a) for the modulation of the laggard and (b) for the modulation of the leader. All plotted rf spectra exhibit the same characteristic structure: an intense low frequency part and smaller peaks corresponding to multiples of $1 /(2 \tau)$. Here, we focus our attention on the peaks at $508 \mathrm{MHz}$, the frequency of the external modulation. Figure 5(a) demonstrates that for modulation of the laggard, the modulation peak is clearly present with comparable intensity in both spectra. Hence, a filtering of the periodic signal does not take place, and the modulation signal is transferred to the leader. However, the situation drastically changes when the leader is modulated. Figure 5(b) demonstrates that the modulation peak, which is dominant in the leader spectrum, is not present in the laggard spectrum; i.e., the laggard acts as a chaos pass filter and suppresses the periodic signal. Thus, we demonstrate chaos synchronization in delay-coupled SLs: the leader indeed synchronizes the laggard. We point out that this behavior is symmetric under the exchange of leader and laggard roles of the lasers which takes place when the detuning between the two lasers is reversed.

The basic physical mechanism underlying to the coupling-induced instabilities and the associated symmetrybreaking can thus be interpreted as follows: the chaotic signal of the leader synchronizes the laggard, whereas the 


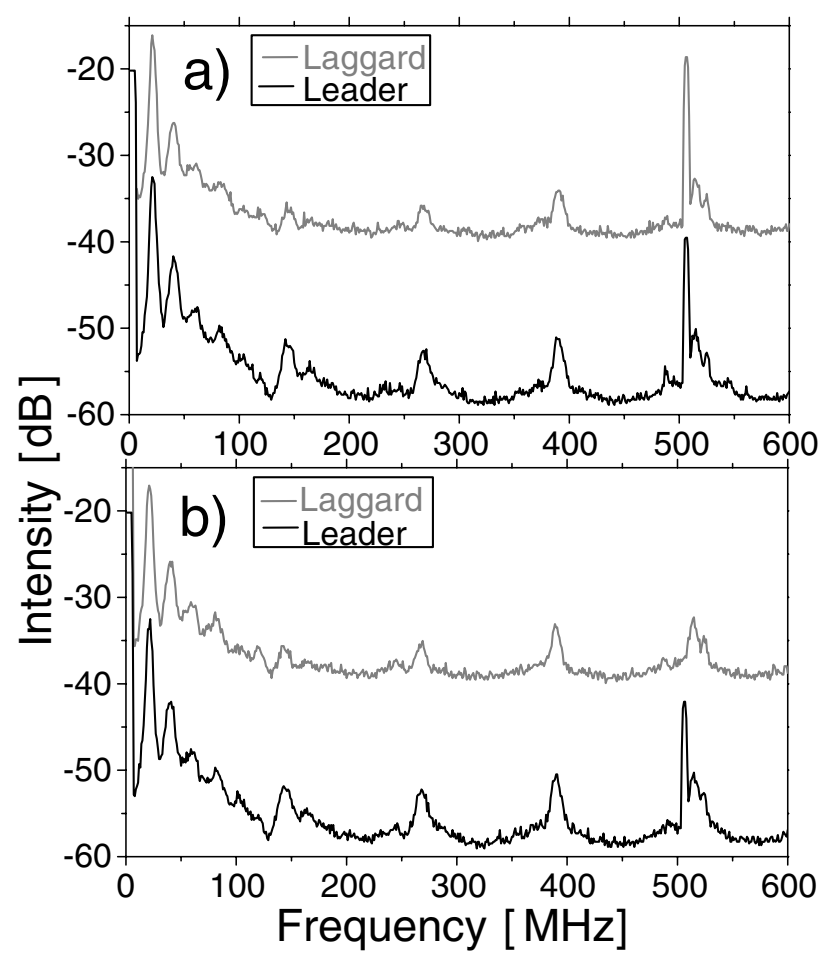

FIG. 5. rf spectra of leader and laggard. (a) Modulation of the laggard. (b) Modulation of the leader. The injection current is $I=I_{\mathrm{th}}^{\text {sol }}$, and the coupling time is $\tau=3.83 \mathrm{~ns}$.

laggard acts as a driving force of the chaotic dynamics. The symmetry-breaking manifests itself in these different physical roles of the subsystems. The fact that the leader synchronizes the laggard obviously results in the synchronous dynamical behavior delayed by the coupling time $\tau$. The driving role of the laggard leads to the observed feedbacklike dynamical behavior: the signal of the leader synchronizes the laggard whose signal, in turn, is injected into the leader with a delay of $2 \tau$ after the emission of the original leader signal. Consequently, the dynamics of the delay-coupled lasers is comparable to the dynamics of a single laser subject to optical feedback with a delay of $2 \tau$, as opposed to $\tau$, which one would expect from simple symmetry upon reflection considerations. A stability analysis promises further insight into the physical mechanisms determining the dynamical behavior of the system. Furthermore, the SL specific origin of synchronization phenomena is still an open question.
To sum up, we have reported on subnanosecond, coupling-induced synchronized chaotic dynamics in conjunction with a spontaneous symmetry-breaking in symmetrically delay-coupled SLs. We have confirmed our experimental results by numerical simulations of two identical subsystems using a rate equation model. We have identified the large coupling time, the symmetry-breaking, and the chaos synchronization as the key elements leading to the observed coupling-induced instabilities. From our results, we conjecture that the coupling-induced instabilities, and the leader-laggard-type conjunction of chaos synchronization with symmetry-breaking are not SL specific but are linked to the delay present in the coupling and could also occur in other delay-coupled nonlinear oscillators.

*Permanent address: Departament de Física, Universitat de les Illes Balears, E-07071 Palma de Mallorca, Spain.

[1] K. Coffman, W. D. McCormick, and H. L. Swinney, Phys. Rev. Lett. 56, 999 (1986); S. H. Strogatz and I. Stewart, Sci. Am. 269, No. 12, 68 (1993); C. Schäfer, M. G. Rosenblum, J. Kurths, and H. H. Abel, Nature (London) 392, 239 (1998); U. Ernst, K. Pawelzik, and T. Geisel, Phys. Rev. Lett. 74, 1570 (1995); R. Roy and K. S. Thornburg, Jr., Phys. Rev. Lett. 72, 2009 (1994); T. Sugawara, M. Tachikawa, T. Tsukamoto, and T. Shimizu, Phys. Rev. Lett. 72, 3502 (1994).

[2] M. K. S. Yeung and S. H. Strogatz, Phys. Rev. Lett. 82, 648 (1999).

[3] S. Kim, S. H. Park, and H. B. Pyo, Phys. Rev. Lett. 82, 1620 (1999).

[4] A. Hohl, A. Gavrielides, T. Erneux, and V. Kovanis, Phys. Rev. Lett. 78, 4745 (1997).

[5] G. D. VanWiggeren and R. Roy, Science 279, 1198 (1998); C. R. Mirasso, P. Colet, and P. Garcia-Fernandez, IEEE Photonics Technol. Lett. 8, 299 (1996); J. P. Goedgebuer, L. Larger, and H. Porte, Phys. Rev. Lett. 80, 2249 (1998).

[6] I. Fischer, Y. Liu, and P. Davis, Phys. Rev. A 62, 011801(R) (2000).

[7] I. Fischer, G. H. M. van Tartwijk, A. M. Levine, W. Elsässer, E. O. Göbel, and D. Lenstra, Phys. Rev. Lett. 76, 220 (1996).

[8] M. G. Rosenblum, A. S. Pikovsky, and J. Kurths, Phys. Rev. Lett. 78, 4193 (1997).

[9] V. Kovanis, A. Gavrielides, T. B. Simpson, and J. M. Liu, Appl. Phys. Lett. 67, 2780 (1995). 